\title{
The taxonomic status of Petropedetes newtonii (Amphibia, Anura, Petropedetidae)
}

\author{
Alberto Sánchez-Vialas', Marta Calvo-Revuelta', \\ Santiago Castroviejo-Fisher², Ignacio De la Riva'
}

I Museo Nacional de Ciencias Naturales (MNCN-CSIC), cl José Gutiérrez Abascal 2, 28006, Madrid, Spain 2 PUCRS, Pontificia Universidade Católica do Rio Grande do Sul, Av. Ipiranga 6681, 90619-900, Porto Alegre, Brazil

Corresponding author: Alberto Sánchez-Vialas (albertosv@mncn.csic.es)

Academic editor: A. Obler | Received 1 March 2018 | Accepted 27 April 2018 | Published 6 June 2018

http://zoobank.org/A6572ECF-3B4B-4014-9B7F-D84B2C4F348B

Citation: Sánchez-Vialas A, Calvo-Revuelta M, Castroviejo-Fisher S, De la Riva I (2018) The taxonomic status of Petropedetes newtonii (Amphibia, Anura, Petropedetidae). ZooKeys 765: 59-78. https://doi.org/10.3897/ zookeys.765.24764

\begin{abstract}
The taxon Petropedetes newtonii was described in 1895 by Bocage, from Bioko Island (Equatorial Guinea). This taxon, whose holotype is lost, has been misidentified since Boulenger's revision of the genus in 1900 and its relationships with other taxa (P. vulpiae and P. johnstoni) is confusing. Currently, P. newtonii is considered a synonym of $P$. johnstoni. In this work, by revising morphological characters of non-webbed Petropedetes of Bioko, we demonstrate the morphological singularity of these specimens with respect to $P$. johnstoni and $P$. vulpiae and their association with the name Petropedetes newtonii. Consequently, we provide the subsequent designation of a neotype of $P$. newtonii and revalidate this species from its synonym with $P$. johnstoni.
\end{abstract}

\section{Keywords}

Bioko, Cameroon, Equatorial Guinea, morphology, neotype, Petropedetes johnstoni, Petropedetes vulpiae, Petropedetes newtonii, taxonomy 


\section{Introduction}

The family Petropedetidae Noble, 1931 includes three genera allopatrically distributed, Arthroleptides Nieden, 1911, from East Africa, the monotypic Ericabatrachus Largen, 1991, from Ethiopia, and Petropedetes Reichenow, 1874, from Central Africa (Frost 2018).

Petropedetes is distributed throughout the Gulf of Guinea, in Western Central Africa, including the island of Bioko, where the species generally inhabit the surroundings of fast-flowing streams. Reproduction takes place in land and male parental care has been described (Barej et al. 2010). Tadpoles generally present a semi-terrestrial life stage, developing in the water film covering the surface of rocks or in the water of running streams (De la Riva 1994, Barej et al. 2010). Eight species are currently recognised within the genus, namely: $P$. cameronensis Reichenow, 1874, P. euskircheni Barej, Rödel, Gonwouo, Pauwels, Böhme \& Schmitz, 2010, P. johnstoni (Boulenger, 1888), P. juliawurstnerae Barej, Rödel, Gonwouo, Pauwels, Böhme \& Schmitz, 2010, P. palmipes Boulenger, 1905, P. parkeri Amiet, 1983, P. perreti Amiet, 1973 and P. vulpiae Barej, Rödel, Gonwouo, Pauwels, Böhme \& Schmitz, 2010. They are generally easy to distinguish from each other based on the development of webbing and tympanum, and in some dimorphic characters of males such as femoral glands, tympanum size, presence and position of tympanic papilla, and skin keratinised spicules (Barej et al. 2010).

The phylogenetic relationships of the genus Petropedetes have been recently revised by Barej et al. (2014), revealing that the species diversity of the genus is underestimated and also that the validity of the taxon P. newtonii (Bocage, 1895), placed in the synonymy of P. johnstoni by Barej et al. (2010), remains uncertain.

Bocage described $P$. newtonii in 1895 as Tympanoceros newtonii. Its description was based on an adult male specimen from Bioko (formerly Fernando Poo) (type locality: "Lîle de Fernão do Pó dans le golfe de Guiné") (Bocage 1895a). The holotype of $P$. newtonii is lost (Perret 1976, Barej et al. 2010) but, fortunately, a detailed illustration and an additional description were published based on a second male specimen collected from Basilé, Bioko (Bocage 1895b). Previous to the description by Bocage (1895a), two more species of Petropedetes had already been described: P. cameronensis Reichenow, 1874 (type species of the genus) and P. johnstoni (= Cornufer johnstoni Boulenger, 1888). Boulenger (1900) transferred Tympanoceros newtonii (misspelled "newtoni") and Cornufer johnstoni to the genus Petropedetes and provided the first revision of the genus, with a synthesis of diagnosable characters for the species recognised at that time (P. cameronensis, P. johnstoni, and P. newtonii). The specimens of "P. newtonii" used by Boulenger (1900) were from mainland Cameroon and no specimens were included from Bioko (type locality of $P$. newtonii). The morphological characters considered in Boulenger's revision to characterise $P$. newtonii were not consistent with the holotype description made by Bocage (1895a). Furthermore, Amiet (1983), following Boulenger's description, studied more characters than those present in the original description of $P$. newtonii (e.g., relative position of tympanic papilla and femoral gland size). Boulenger's (1900) and Amiet's (1983) descriptions of $P$. newtonii were widely used by subsequent authors (Mertens 1968, De la Riva 1994, Lasso et al. 2002, Frétey 
et al. 2012). Incongruences between the original description of $P$. newtonii and the ones using mainland populations (Boulenger 1900, Amiet 1983) have been discussed by Barej et al. (2010). According to Barej et al. (2010), the continental populations of formerly considered $P$. newtonii represent a different evolutionary unit, which was described as P. vulpiae. In their revision, Barej et al. (2010) placed Petropedetes newtonii (Bocage, 1895) in the synonymy of P. johnstoni (Boulenger, 1888), due to the apparent absence of morphological differences between both descriptions. Consequently, two species were considered to occur in Bioko, one of them with webbed toes (P. cameronensis) and the other one with non-webbed toes (P. johnstoni) (Barej et al. 2010). Specimens of non-webbed Petropedetes from Bioko studied using molecular data by Barej et al. (2014) were nested with continental P. vulpiae, but forming a clade, together with a few samples from nearby localities in Cameroon, sister to all other samples of $P$. vulpiae (including topotypic specimens). Barej et al. $(2010,2014)$ indicated that the original description of $P$. newtonii (Bocage, 1895a) does not fit with the morphological characters of $P$. vulpiae and, consequently, they kept them as separate taxa (P. newtonii in the synonymy of $P$. johnstoni).

The results of these studies suggest that three independent evolutionary units of Petropedetes might be present in Bioko. One of them, P. cameronensis, is diagnosable based on molecular and morphological characters and easily recognisable by having males with half-webbed toes, a very small tympanum without tympanic papilla, and metacarpal spine absent. The other two units are non-webbed and correspond to (1) the specimens morphologically assignable to $P$. johnstoni (apparently not studied with molecular data), and (2) the specimens treated as $P$. vulpiae based on molecular data (Barej et al. 2014). The problem posed by Biokoan P. johnstoni and P. vulpiae in relation to $P$. newtonii needs to be clarified.

The objective of this work is to solve the systematics of the non-webbed Petropedetes of Bioko by analysing the morphological characters of the available series of specimens from Bioko included in the molecular phylogeny of Barej et al. (2014) assigned by them to $P$. vulpiae. To do this, we compared the morphological characters of these specimens with (A) the original Bocage's (1895a) description of $P$. newtonii, Boulenger's (1888) of P. johnstoni, and Barej's et al. (2010) of P. vulpiae; and (B) continental specimens of $P$. johnstoni included in Barej et al.'s (2014) molecular study. As a result, we demonstrate the morphological singularity of the non-webbed Petropedetes from Bioko with respect to both P. johnstoni and P. vulpiae and the association of these Petropedetes from Bioko with the original $P$. newtonii. Consequently, we revalidate the taxon $P$. newtonii from its junior synonym with $P$. johnstoni and designate and describe a neotype of $P$. newtonii to allow its fully taxonomic recognition and facilitate future work on Petropedetes.

\section{Materials and methods}

We revised 14 specimens of non-webbed Petropedetes from several localities of Bioko (Equatorial Guinea) held at the herpetological collection of the Museo Nacional de 
Ciencias Naturales (MNCN-CSIC), Madrid, Spain. Three specimens (MNCN 46703, MNCN 46708, and MNCN 46719) were collected in March 2007 and preserved in $70 \%$ ethanol. The other 11 specimens were collected in November and December 2003, fixed in formalin $10 \%$ and preserved in ethanol $70 \%$. Prior to fixation, a piece of tissue was preserved in ethanol $96 \%$ and stored in a freezer for molecular studies. Among them, three specimens were previously included in the molecular analysis of Barej et al. (2014): MNCN 48728 (MNCN-DNA 50405), MNCN 48729 (MNCN-DNA 50465) and MNCN 48730 (MNCN-DNA 50411). Additional specimens of Petropedetes johnstoni stored in $70 \%$ ethanol and housed at the Zoologisches Forschungsmuseum Alexander Koenig (ZFMK 87709, adult male, and ZFMK 87710, adult female) were studied. These specimens were collected from Nkoelon, Campo region $\left(2^{\circ} 23^{\prime} 49.8^{\prime \prime N}, 10^{\circ} 02^{\prime} 47.4^{\prime \prime E}\right)$, Cameroon. Studied specimens and their associated data are listed in Table 1. Nomenclature used in the morphological description of the neotype follows De la Riva et al. (2012).

Measurements were taken with a digital caliper to the nearest $0.1 \mathrm{~mm}$, and are given in $\mathrm{mm}$. Morphometric abbreviations are as follows:

\author{
SVL (snout-vent length) \\ HL (head length, from rictus to point \\ of snout) \\ HW (head width, at level of rictus) \\ IND (internarial distance) \\ END (distance from eye to nostril) \\ TD (horizontal tympanum diameter) \\ ED (eye diameter) \\ NS (distance from nostril to snout tip)
}

FL (femur length)

FGL (femoral gland length)

FGW (femoral gland width)

TL (tibia length)

FTL (foot length, from proximal border of inner metatarsal tubercle to tip of fourth toe)

THL (thenar tubercle length)

THBL (thumb length)

For qualitative morphological diagnosis, we selected male specimens, which possess the most important characters to differentiate these species. These features are: tympanum size, presence of tympanic papilla and its relative position in the tympanum, presence of keratinised spicules on basis of arms, relative size of femoral glands and webbing development.

Digital photographs were taken with a reflex camera fitted with a macro lens. Micro-computed tomography (micro-CT) scans were carried out for two specimens (male and female) of non-webbed Petropedetes from Bioko (MNCN 48728, MNCN 48729) and in the same way for two specimens (male and female) of P. johnstoni (ZFMK 87709, ZFMK 87710), at the MNCN. The scans were produced using a XTH 160 Nikon Metrology, with a molybdenum target. Specimens were scanned with the following settings: $126 \mathrm{kV}$, and $47 \mu \mathrm{A}$ over 1000 projections during 1.30 h. Raw X-ray data were processed using CTPro 3D software (Nikon Metrology) and micro-CT images were analysed using VG Studio MAX 3.0.3 (Volume Graphics, Heidelberg, Germany). 


\begin{tabular}{|c|c|c|c|c|c|c|c|c|c|c|c|c|c|c|c|c|c|}
\hline II 9 & $\bar{\Xi}$ & త్రి & i & $\exists$ & \pm & $\stackrel{\sim}{\sim}$ & $\triangleq$ & & $\exists$ & & 5 & ن & in & ?. & 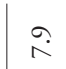 & $\hat{i}$ & \\
\hline & 竞 & & & & $\stackrel{\Downarrow}{ \pm}$ & & & & & & $\mathbb{\sigma}^{\circ}$ & & $?$ & & & & \\
\hline & 离 & 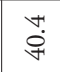 & & & $\begin{array}{l}\tilde{n} \\
\tilde{n} \\
\tilde{n}\end{array}$ & & & & $\hat{\tilde{\lambda}}$ & & \pm & & & & & $\widetilde{N}$ & \\
\hline 窇焉 & ڤ్ & 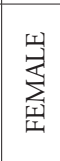 & $\begin{array}{c}\stackrel{5}{E} \\
\text { 空 } \\
\text { Z }\end{array}$ & 8 & 恶 & & 突 & 島 & 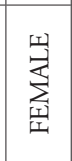 & & 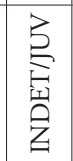 & 总 & 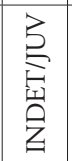 & & $\vec{r}$ & 宗 & \\
\hline ह & 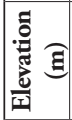 & $\infty$ & $\infty$ & i & 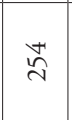 & & $\hat{\approx}$ & & तิ & & $\hat{\imath}$ & & & & $\hat{\imath}$ & $\exists$ & \\
\hline$\hat{\overrightarrow{\vec{E}}}$ & 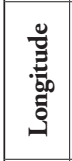 & 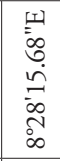 & 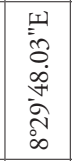 & 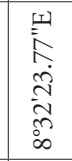 & 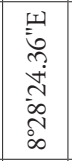 & సิ & 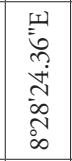 & ले & 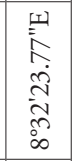 & 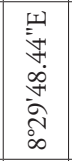 & 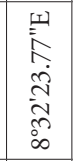 & 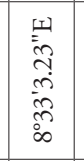 & & & 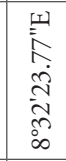 & 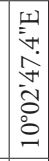 & \\
\hline 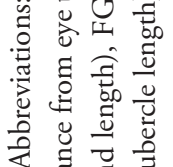 & 㺼 & 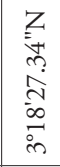 & 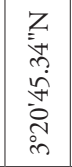 & 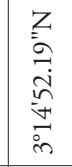 & 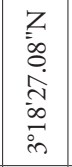 & $c^{3}$ & & 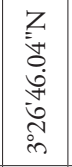 & $\begin{array}{l}Z \\
\bar{Z} \\
\vec{i} \\
\end{array}$ & & $\equiv$ & & & & 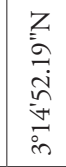 & 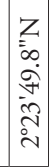 & \\
\hline 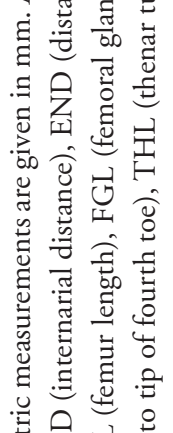 & 胥 & 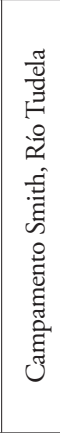 & 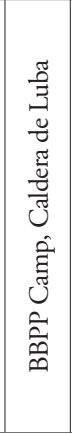 & 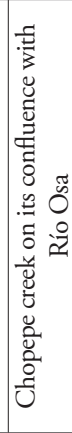 & 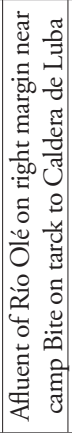 & 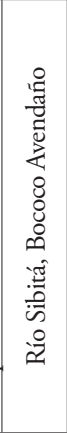 & 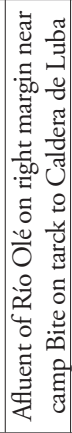 & 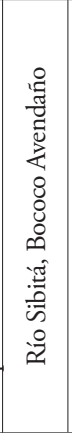 & 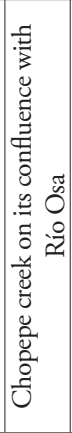 & 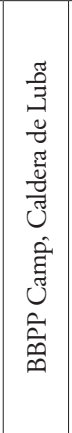 & 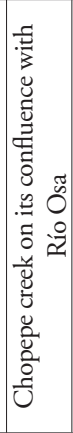 & 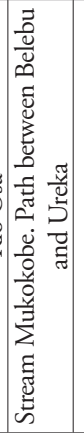 & 更 & 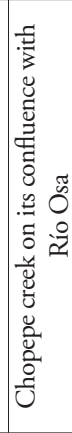 & 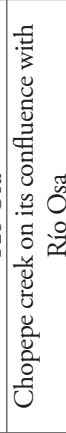 & 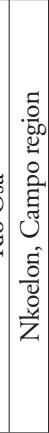 & \\
\hline 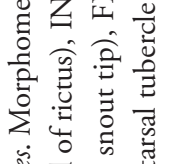 & 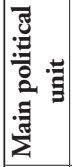 & $\begin{array}{l}0 \\
\frac{\pi}{a} \\
0 \\
0 \\
0 \\
0\end{array}$ & 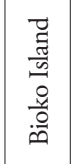 & 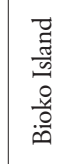 & 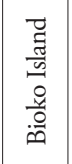 & 7 & 告 & 6 & 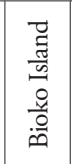 & 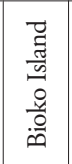 & 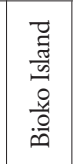 & 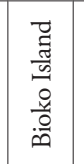 & a & 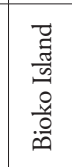 & 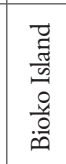 & 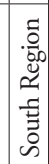 & \\
\hline$\vec{\sigma}$ & 总 & 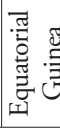 & & . & & & & & & & 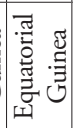 & 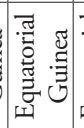 & 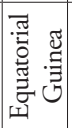 & 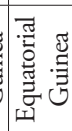 & & స్ & \\
\hline 4 & 플 & ç & 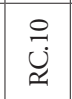 & $\begin{array}{l}n \\
\mathfrak{c} \\
\mathfrak{c}\end{array}$ & $\stackrel{n}{3}$ & & ร & & 合 & & 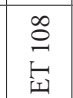 & & & & & 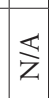 & \\
\hline $\mathrm{Z}$ & 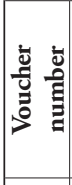 & 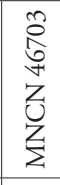 & 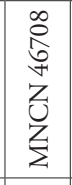 & 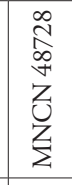 & 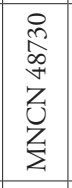 & ह & $\begin{array}{l}y \\
y \\
a \\
0 \\
2 \\
z \\
y\end{array}$ & 2 & 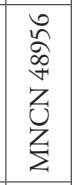 & 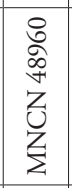 & $\stackrel{g}{g}$ & 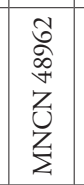 & $\begin{array}{l}2 \\
\hat{\mathbb{S}} \\
+ \\
z \\
z \\
z \\
z\end{array}$ & & Z & $\begin{array}{l}a \\
\hat{p} \\
\infty \\
\dot{\infty} \\
\sum_{x}\end{array}$ & \\
\hline$\dot{ }$ & .్ֶ & 离 & 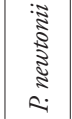 & 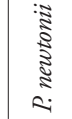 & : & 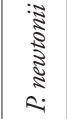 & 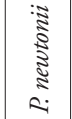 & 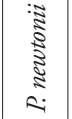 & 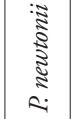 & : & 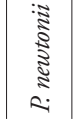 & 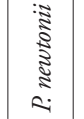 & 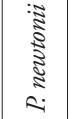 & : & 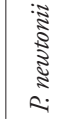 & $?$ & \\
\hline
\end{tabular}




\begin{tabular}{|c|c|c|c|c|c|c|c|c|c|c|c|c|c|c|}
\hline 8 & $\mathbb{Z} \mathbb{Z}$ & פ̆ & & 盛 & 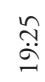 & $\begin{array}{l}\text { fo } \\
\dot{\infty}\end{array}$ & 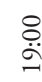 & 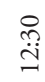 & ֻू & & & 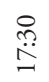 & : & $\vec{z}$ \\
\hline$\overline{0}^{\circ}$ & 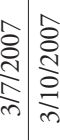 & 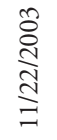 & 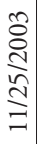 & 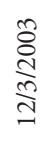 & 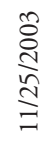 & 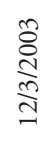 & 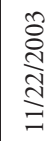 & 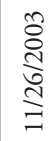 & $\begin{array}{l}\tilde{\vdots} \\
\vdots \\
\vdots \\
\vdots\end{array}$ & & 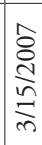 & 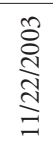 & 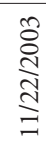 & \\
\hline & & 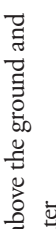 & 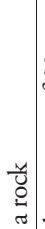 & 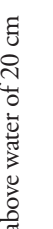 & 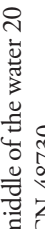 & 2 & 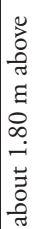 & 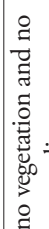 & 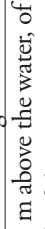 & & & 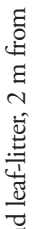 & 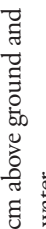 & \\
\hline
\end{tabular}

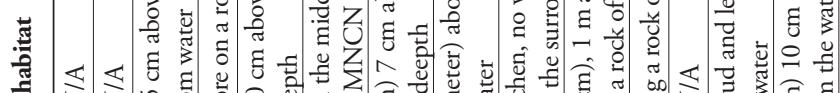

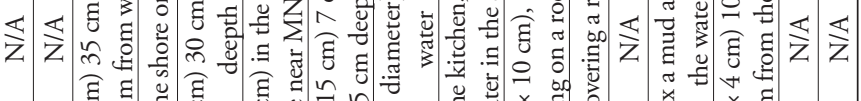

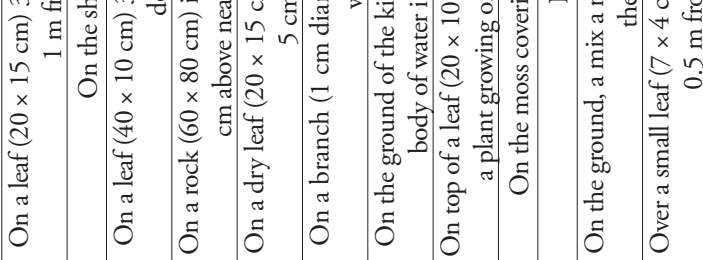

盖

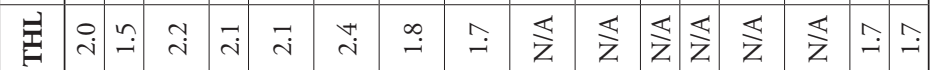

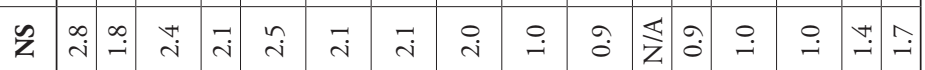

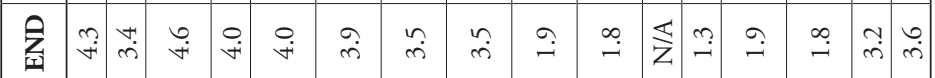

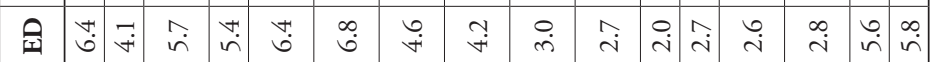

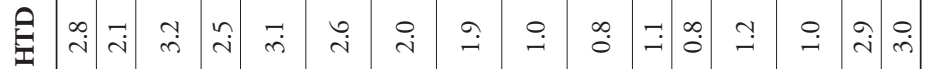

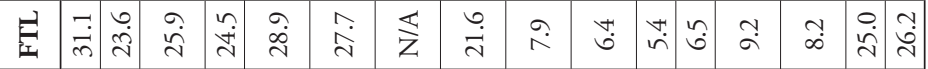

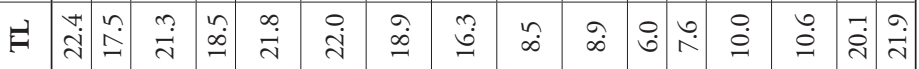

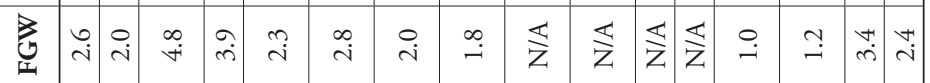

矛

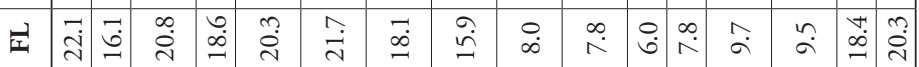

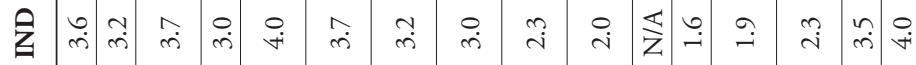

\begin{tabular}{|c|c|c|c|c|c|c|c|c|c|c|c|c|c|}
\hline & 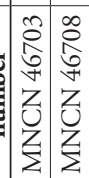 & 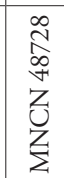 & $\mid \begin{array}{c}0 \\
\infty \\
\infty \\
0 \\
z \\
0 \\
z \\
z \\
\Sigma\end{array}$ & 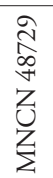 & 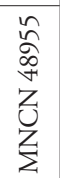 & $\begin{array}{l}\hat{n} \\
\text { ò } \\
\dot{+} \\
z \\
\vdots \\
z \\
z\end{array}$ & 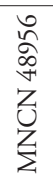 & 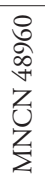 & 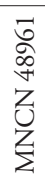 & 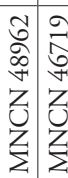 & 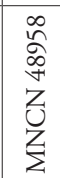 & $\begin{array}{l}\text { à } \\
\alpha \\
\alpha \\
z \\
z \\
z \\
z\end{array}$ & \\
\hline & 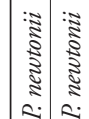 & 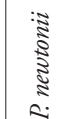 & | & $\begin{array}{l}\text { ב } \\
\text { s. }\end{array}$ & 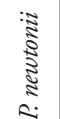 & 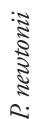 & 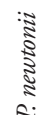 & 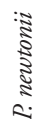 & 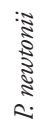 & & 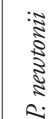 & & \\
\hline
\end{tabular}




\section{Results and discussion}

\section{Petropedetes johnstoni and P. newtonii original descriptions}

Petropedetes johnstoni was described by Boulenger in 1888, based on a subadult specimen from Río del Rey, Cameroon (“Cameroons") (= river Ndian, Western Cameroon). Despite it was treated as a female by Boulenger (1888), Parker's (1936) revision of the holotype stated that it corresponds to an immature male (Amiet 1983).

Bocage (1895a) described Petropedetes newtonii from Bioko based on a single adult male specimen with well-marked sexual features.

We agree with Barej et al. (2010), which stated that morphological discrepancies between both descriptions are due to ontogeny, measurement methods (as for the tympanum size), and intraspecific variability (as for the relative position of the tibiotarsal articulation in regard to the snout tip when the leg is stretched forwards). While the holotype of P. johnstoni is a subadult male which lacks the typical male secondary characters, the holotype description of $P$. newtonii resembles that of a male possessing reproductive features. Barej et al. (2010) argue that both descriptions represent morphologically indistinguishable taxa and, with the evidence at hand, considered $P$. newtonii a junior synonym of $P$. johnstoni. However, we argue that this decision needs to be evaluated in the light of detailed comparisons of adult male specimens, which requires, for reasons outlined above, the study of additional specimens besides the type series. These results are presented in the following sections.

\section{Morphological revision of $P$. johnstoni}

Two specimens of P. johnstoni (ZFMK 87709, ZFMK 87710) were morphologically revised in order to complete the original description of the holotype made by Boulenger (1888). The selected specimens were previously characterised based on molecular data by Barej et al. (2014) and are unambiguously nested in the clade of P. johnstoni, which includes samples from the type locality of P. johnstoni.

Both specimens are characterised by: whitish posterior side of the thighs with a speckled pattern made up of brownish dots or marks (Fig. 1); ventral side whitish; lower jaw rounded, with several well marked white spots; snout slightly rounded in dorsal and ventral view (Figs 1, 2A, B); relatively small vomerine teeth (Fig. 3B); supratympanic fold distinct (Fig. 4A); palmar tubercle oval (Fig. 5B); thenar tubercles in both specimens oval (Fig. 5B), approximately $1 / 4$ of total length of Finger I; one subarticular tubercle on Finger I, placed between fingertip and centre of the finger; subarticular tubercle of Finger II centrally positioned on the finger; fingers III and IV with two subarticular tubercles; fingertips with adhesive discs of different sizes, as follows: I > II > III = IV; relative lengths of fingers: III > IV > II > I; toes not webbed or rudimentarily; outer metatarsal tubercle absent; inner metatarsal tubercle elongated, at the base of Toe I. Toes I and II with one single tubercle, toes III and V with two single tubercles and Toe III with three single tubercles; no supernumerary tubercles; relative 

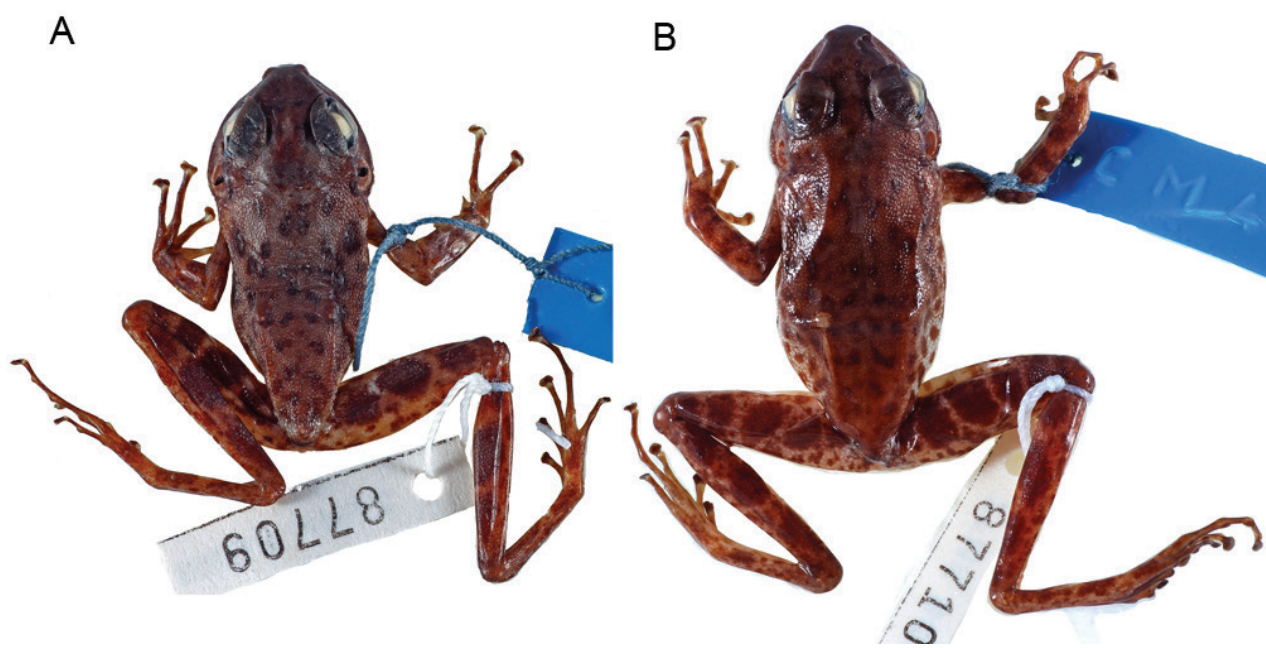

Figure I. Dorsal view of Petropedetes johnstoni specimens (A male, ZFMK 87709 B female, ZFMK 87710).

lengths of toes: IV $>$ III $>$ V > II > I; skin with relatively small warts, especially on the anterior part of dorsum. Morphometric measurements are given in Table 1.

Male specimen ZFMK 87709 is characterised by possessing a small tympanum (relation of the TD to $\mathrm{ED}=0.53$ ), tympanic papilla present in the upper border of the tympanum (Fig. 4A), a big femoral gland covering most of the femoral skin (relation of femoral gland to femur length $=0.43$; Fig. $2 \mathrm{~A}$ ), lack of keratinised spicules on the skin of the basis of arms (Fig. 2A), upper and lower border of tympanum round, not flattened (Fig. 4A), forearm not hypertrophied (Fig. 2A), metacarpal spines absent (Fig. 6A) and webbing rudimentary.

The study of the humerus by CT-scan analyses of a male (ZFMK 87709; Fig. 7A) and a female (ZFMK 87710) of P. johnstoni, shows a strong sexual dimorphism due to the presence in the male of a double crest, relatively short in length and distally divergent in the dorsal side of the bone, which is totally absent in the female.

\section{Summary of the diagnostic characters of $P$. vulpiae}

The other species involved in the taxonomic problem of Petropedetes newtonii, and recently suggested to be in Bioko on the basis of DNA sequences (Barej et al. 2014), is $P$. vulpiae. Before we proceed with a description of the morphology of the new material of non-webbed Petropedetes from Bioko, we summarised the main characters of this species to allow a clearer discussion. The diagnosis made by Barej et al. (2010) reads as follows: "medium sized Petropedetes; compact body shape; tympanum usually flattened on the upper and lower border; tympanum larger than diameter of eye in males, smaller in females; characters of breeding males: tympanic papilla present (broad, fleshy), papilla closer to the centre than the upper border; forearm hypertrophy well developed; carpal 
A
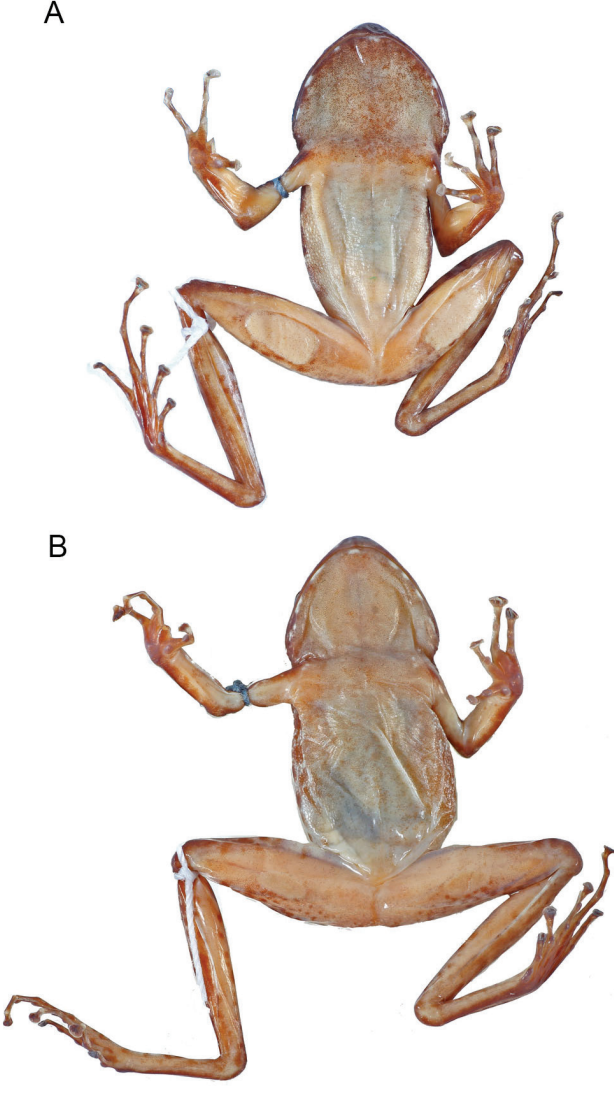

C

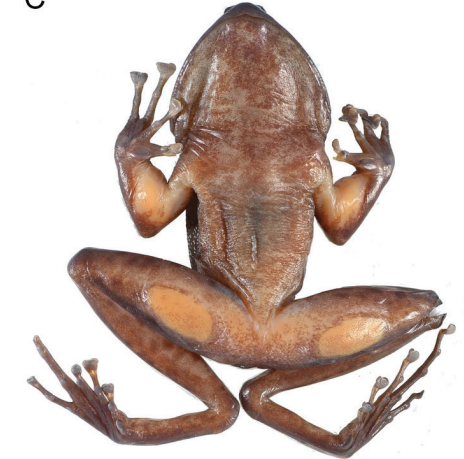

D

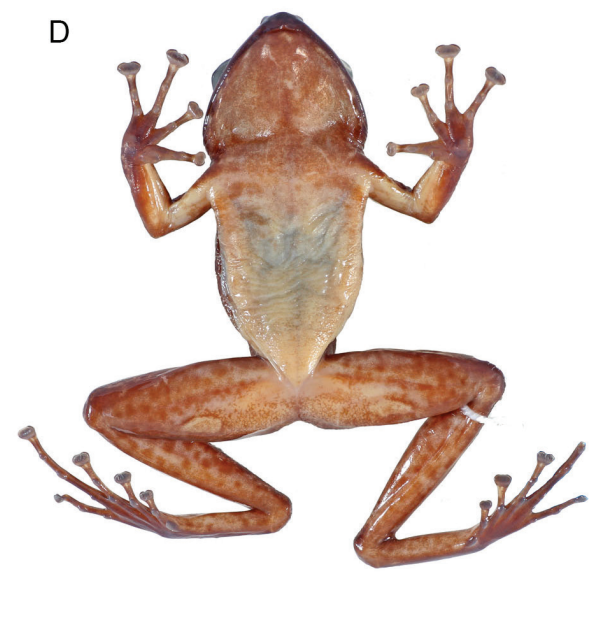

Figure 2. Ventral view of specimens of Petropedetes johnstoni (A male, ZFMK 87709 B female, ZFMK 87710) and P. newtonii (C male, MNCN 48730 D female, MNCN 48729).

spike present; spinosities on throat, forearms and on almost every wart on flanks and dorsum, even around the tympanum; femoral glands large, very prominent; webbing rudimentary". Also, comparative drawings of some features as femoral glands and tympanum of P. vulpiae were included in Barej et al.'s (2010) revision, illustrating the most relevant characters and allowing indirect observations.

\section{Description of the new material of non-webbed Petropedetes from Bioko}

We revised the morphology of a series of 14 specimens (8 juveniles, 4 adult females, 2 adult males) collected in Bioko, including the three individuals studied in the molecular phylogeny of Barej et al. (2014). The adults have the following characteristics: Lower jaw relatively triangular; snout slightly pointed in dorsal and ventral view (Figs 2C, D, 8); relatively big vomerine teeth (Fig. 3A); supratympanic fold distinct 


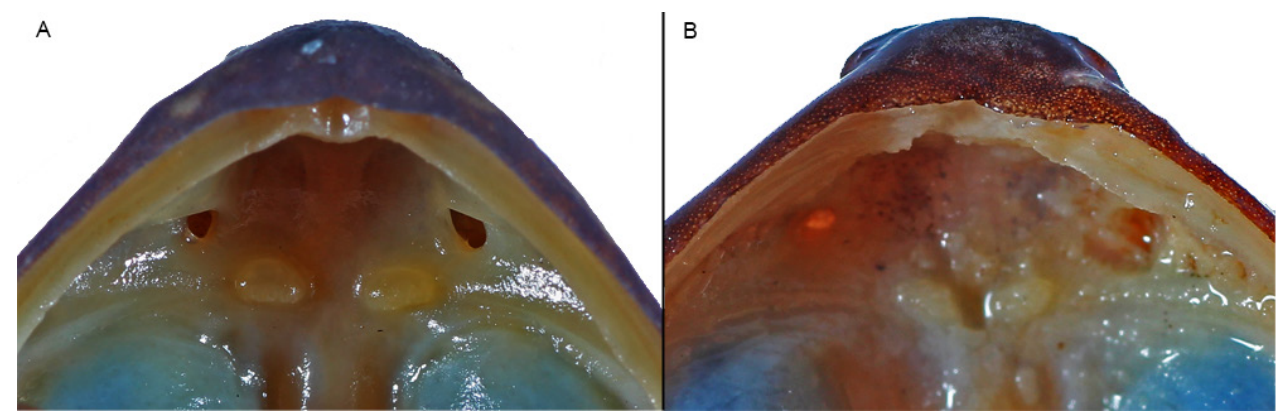

Figure 3. Mouth roof region of Petropedetes newtonii (A female, MNCN 48729) and Petropedetes johnstoni (B male, ZFMK 87709).

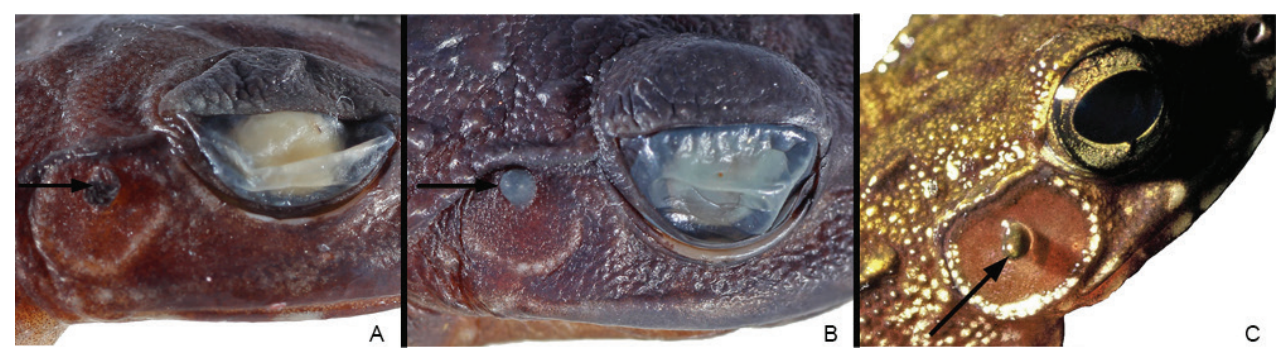

Figure 4. Details of the tympanum of Petropedetes johnstoni (A male, ZFMK 87709), P. newtonii (B male, MNCN 48728), and P. vulpiae (C male, non-collected specimen from Río Muni, Equatorial Guinea; photo by Ignacio De la Riva).

(Fig. 4B); palmar tubercle present, oval (Fig. 9). Thenar tubercle elongated, more than 1/3 of Finger I total length (Figs 5A, 9); one subarticular tubercle on Finger I, placed between fingertip and the centre of the finger; subarticular tubercle of Finger II centrally positioned on the finger; fingers III and IV with two subarticular tubercles, fingertips with adhesive discs of different sizes, as follows: I > II > III = IV; relative lengths of fingers: III > IV > II > I; toes not webbed or rudimentarily; outer metatarsal tubercle absent; inner metatarsal tubercle elongated, on the base of Toe I. Toes I and II with one single tubercle, toes III and V with two single tubercles and Toe III with three single tubercles; no supernumerary tubercles; relative lengths of toes: IV $>$ III $>$ V > II > I. Skin with relatively medium-sized warts, especially on the anterior part of dorsum. Morphometric measurements are given in Table 1, including two grown juveniles.

Both adult male specimens (MNCN 48728 and 48730) are characterised by sharing the following features: (1) the size of the tympanum (relation between TD and ED $=0.56$ and $0.46 \mathrm{~mm}$ respectively), which is approximately half size of the eye diameter; (2) the position of the tympanic papilla in the upper border of tympanum (Fig. 4B); (3) the tympanum upper and lower borders round, not flattened; (4) the relatively large femoral glands (Figs 2C, 8), covering a big area of the femoral skin (relation of femoral gland to femur length $=0.33$ and 0.38 , respectively); (5) the presence of 


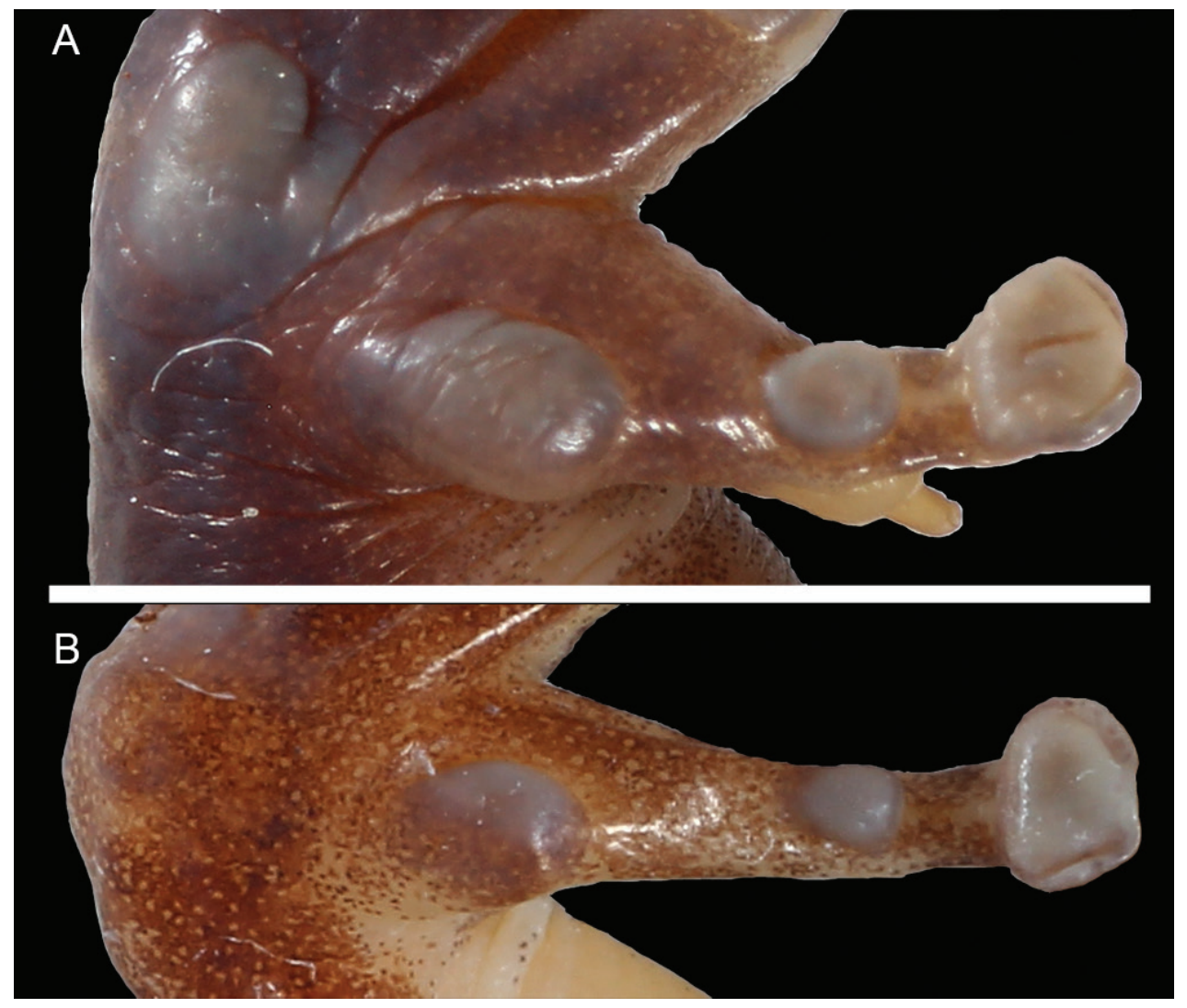

Figure 5. Thenar tubercle of males of Petropedetes newtonii (A male, MNCN 48728) and P. johnstoni (B male, ZFMK 87709). Note the different shape and size and the presence of a dorsal spine on the distal edge of the metacarpal of Finger I in P. newtonii.

dispersed keratinised spicules on the skin of the basis of forelimb, inner surface of upper arm, lower tympanic region and supratympanic fold, and on the postcommissural region (more distinct in MNCN 48728; Fig. 9); (6) the hypertrophied, well developed forelimb (Figs 8, 9); (7) the dorsal spine on the distal edge of the metacarpal of Finger I (Figs 5A, 6B, 9); and (8) the rudimentary webbing (Fig. 9).

The study of the humerus by CT-scan of a male (MNCN 48728; Fig. 7B) and a female (MNCN 48729) shows a strong sexual dimorphism due to the presence in the male of a well-developed double crest, distally divergent in the dorsal side of the bone, which is totally absent in the female.

\section{Comparison of P. johnstoni, P. vulpiae, and non-webbed Petropedetes from Bioko}

Considering the descriptions provided above, the specimens from Bioko could represent: (i) a new species yet to be named under the rules of the ICZN; (ii) part of $P$. 


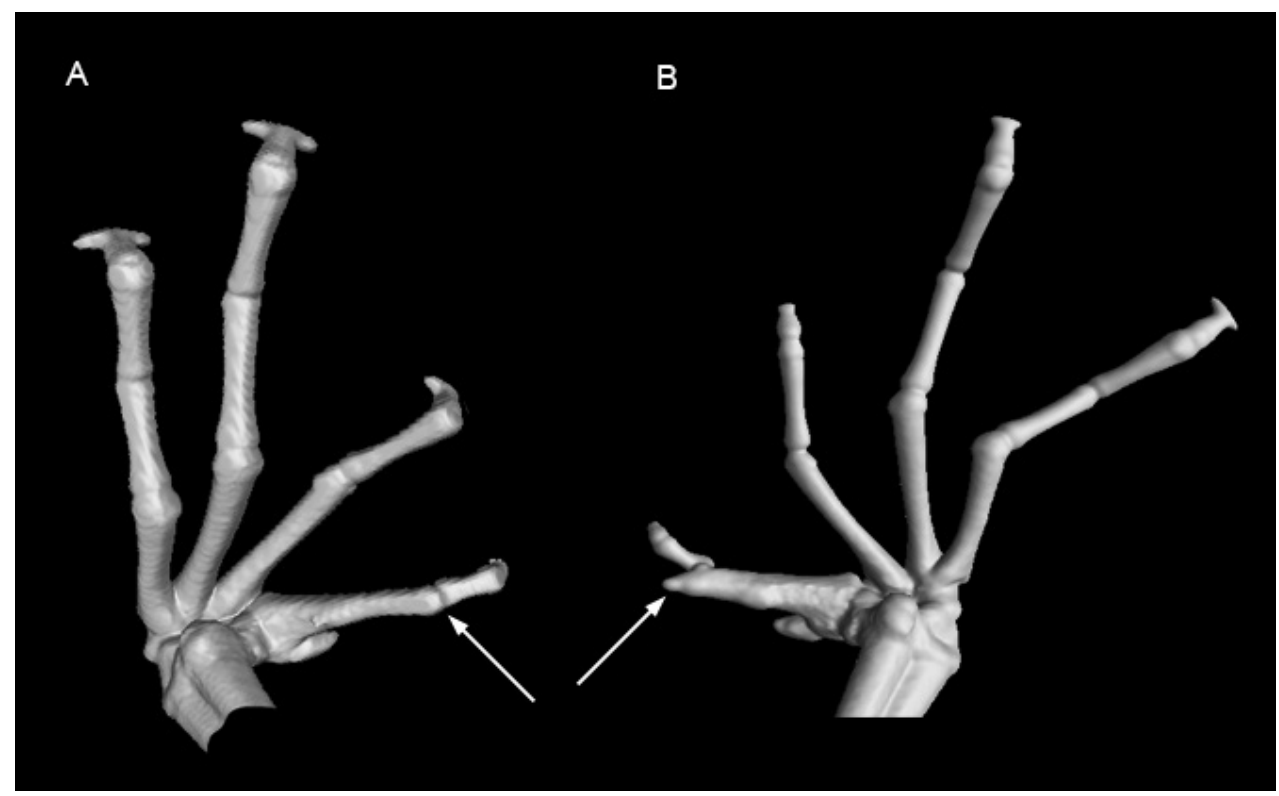

Figure 6. CT-scan image of the dorsal side of the hands of Petropedetes johnstoni (A male, ZFMK 87709) and $P$. newtonii (B male, MNCN 48728). No traces of metacarpal spines are detected in P. johnstoni.

vulpiae as suggested by the phylogenetic analyses of DNA sequences by Barej et al. (2014); (iii) part of $P$. newtonii as a synonym of $P$. johnstoni; or (iv) part of $P$. newtonii as a valid species. We argue in favour of the last scenario.

Our description of non-webbed Petropedetes from Bioko is fully concordant with the description of $P$. newtonii. In other words, none of the characters described in the original description of $P$. newtonii is incompatible with our own observations. Considering the geographic relationship between the specimens (both from Bioko) and their morphological similarity, we consider these specimens part of $P$. newtonii. Furthermore, our detailed study of external and internal morphology of specimens of both $P$. johnstoni and $P$. newtonii led us to discover a number of important differences (Table 2): (i) absence of keratinised spicules on the skin of the throat and on the basis of the arms in P. johnstoni (see also Amiet 1983), which are present in P. newtonii; (ii) absence of metacarpal spines in the adult male of the revisited specimen of $P$. johnstoni (Fig. 6), which are present in studied specimens of $P$. newtonii; (iii) specimens of both sexes of $P$. johnstoni present a lower jaw and a snout more rounded than specimens of P. newtonii; (iv) thenar tubercles are oval and distinctly smaller in P. johnstoni than in studied specimens of $P$. newtonii; and (v) the vomerine teeth are smaller in $P$. johnstoni than in studied specimens of $P$. newtonii, and transversally oriented (Fig. 3). Based on these differences, we consider Petropedetes newtonii a valid taxon and revalidate it from its junior synonym with $P$. johnstoni.

Petropedetes vulpiae is easily distinguishable from $P$. johnstoni and $P$. newtonii, sensu this work, based on the sexual dimorphic characters present in reproductive 


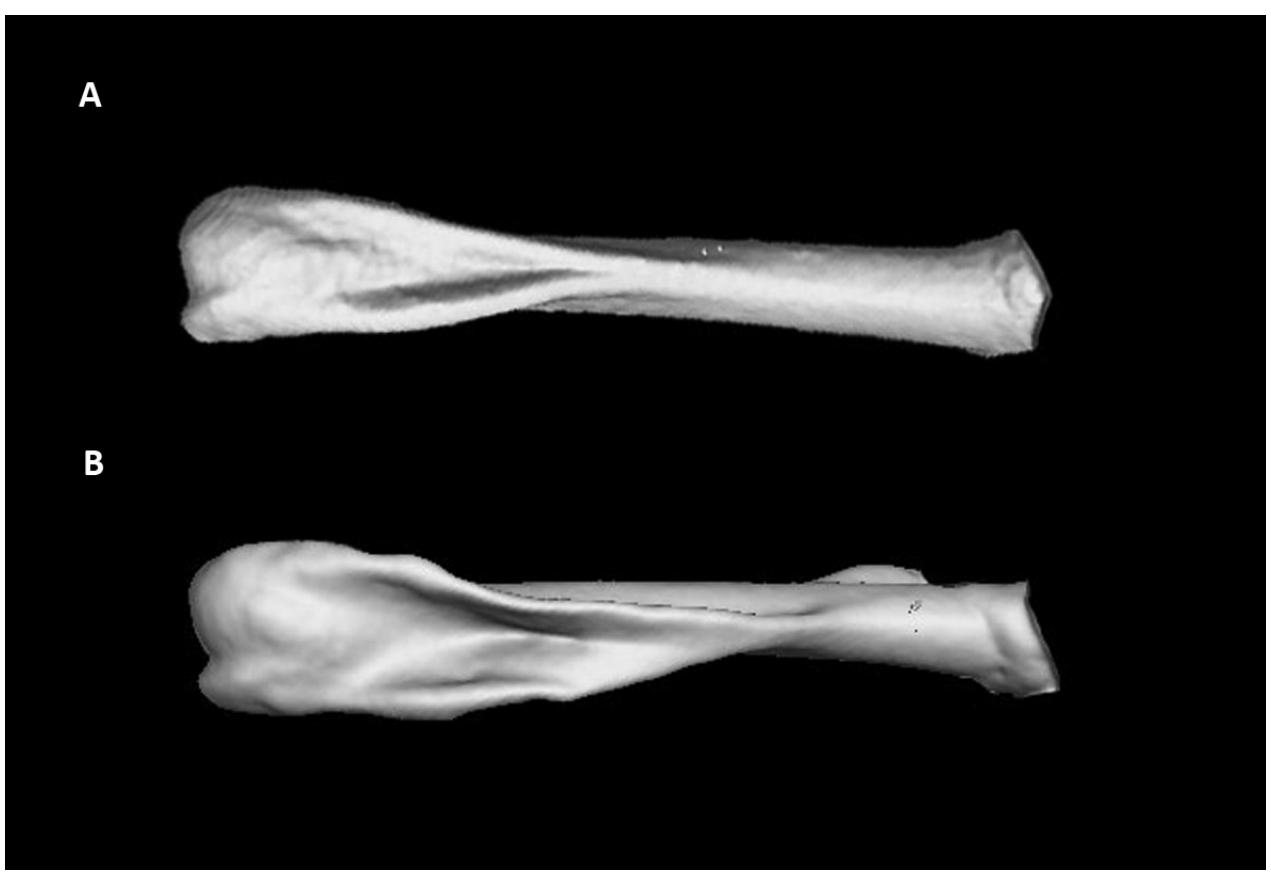

Figure 7.CT-scan image of the dorsal side of the humerus of Petropedetes johnstoni (A male, ZFMK87709) and P. newtonii (B male, MNCN 48728). Note the differences in the development and extension of the bone crest.

Table 2. Morphological data and character states for the studied Petropedetes species.

\begin{tabular}{l|c|c|c}
\hline \multicolumn{1}{c|}{ Species } & $\boldsymbol{P}$ vulpiae & $\boldsymbol{P}$ johnstoni & $\boldsymbol{P}$ newtonii \\
\hline Male tympanum size & $\begin{array}{c}\text { Bigger than eye } \\
\text { diameter }\end{array}$ & Smaller than eye diameter & $\begin{array}{c}\text { Smaller than eye } \\
\text { diameter }\end{array}$ \\
\hline $\begin{array}{l}\text { Tympanic papilla position } \\
\text { Tympanum upper border } \\
\text { shape }\end{array}$ & Close to the centre & Close to upper border & Close to upper border \\
\hline Dorsal metacarpal spine & Present & Rounded & Rounded \\
\hline Skin keratinised spicules & Present & Absent & Present \\
\hline Male humerous crest & Unknown & Relatively short & $\begin{array}{c}\text { Long and well devel- } \\
\text { oped }\end{array}$ \\
\hline Snout shape & Slightly pointed & Slightly rounded & Slightly pointed \\
\hline Thenar tubercle lenght & Unknown & $\begin{array}{c}\text { Shorter than } 1 / 3 \text { of the } \\
\text { finger I }\end{array}$ & $\begin{array}{c}\text { Longer than } 1 / 3 \\
\text { of finger I }\end{array}$ \\
\hline
\end{tabular}

males (Table 2). Petropedetes vulpiae possesses a bigger tympanum, flattened on its upper and lower borders (Fig. 4), and the papilla is closer to the centre of the tympanum. The results of the phylogenetic analyses Barej et al. (2014) are puzzling. The Biokoan specimens studied by us and herein assigned to $P$. newtonii that are included 


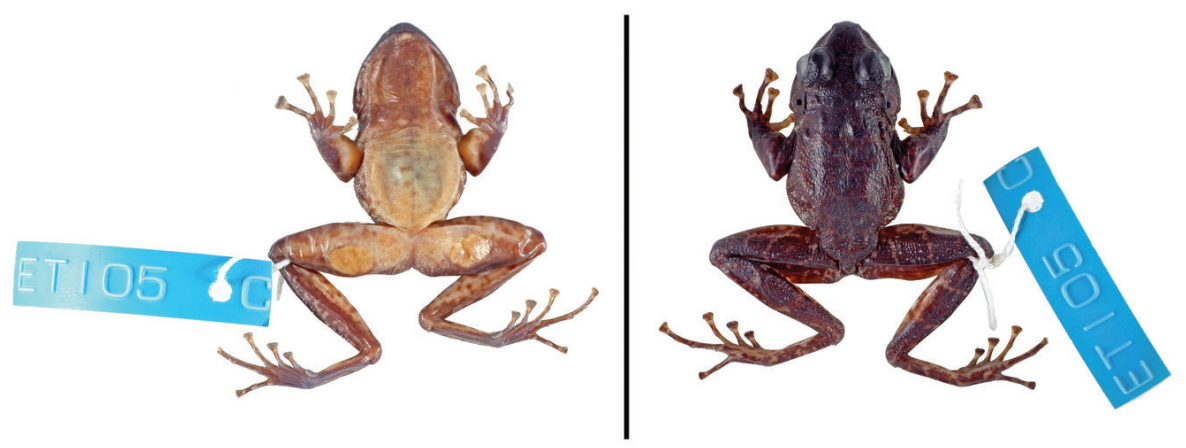

Figure 8. Neotype of Petropedetes newtonii (adult male, MNCN 48728). Left: ventral view; right: dorsal view.

in Barej et al. (2014) cluster with three purported specimens of $P$. vulpiae from nearby continental Cameroon, with nearly identical haplotypes. However, topotypic samples of $P$. vulpiae are located in the sister clade of the Biokoan specimens together with samples from other localities of Cameroon and Nigeria (Barej et al. 2014). To solve the incongruence between the morphological and molecular data at hand, we reidentified the specimens of $P$. vulpiae ZMB 78421, NMP6V 73439/1 and 73439/1 sensu Barej et al. (2014) as $P$. newtonii. Thus, $P$. newtonii and $P$. vulpiae are reciprocally monophyletic sister species.

\section{Designation and description of the neotype of Petropedetes newtonii}

The morphological and molecular distinctiveness of Petropedetes from Bioko in relation to $P$. vulpiae and $P$. johnstoni are clear. As the type material of $P$. newtonii is lost, we deem it necessary to designate a neotype.

Tympanoceros newtonii Bocage, 1895: 270, bona species. Terra typica: "Lîle de Fernão do Pó dans le golfe de Guiné".

Petropedetes newtoni-Boulenger 1900: 439 (misspelled).

Neotype. An adult male in the collection of the Museo Nacional de Ciencias Naturales (Madrid, Spain), MNCN 48728, field number ET105, collected on 22 November 2003 by Santiago Castroviejo-Fisher at Chopepe creek at its confluence with Río Osa (314'52.19"N, 8³2'23.77"E, 27 m a.s.l.), Bioko, Equatorial Guinea (Fig. 9).

Description. Measurements (in $\mathrm{mm}$ ) are listed in Table 1. Adult male specimen of medium size (SVL $34.9 \mathrm{~mm}$ ) in good state of preservation, with distant phalange of Toe IV cut for molecular analyses; body relatively robust; head slightly wider than long (head width $44.4 \%$ of SVL; head length $40.4 \%$ of SVL). Head moderately triangular in shape; snout relatively pointed in dorsal and ventral view; nostrils protuberant, laterally oriented and very close to tip of snout; internarial distance $3.7 \mathrm{~mm}$ (23.9\% of $\mathrm{HW})$; nostrils closer to tip of snout than to margin of orbit; internarial region slightly concave; eye very large, 


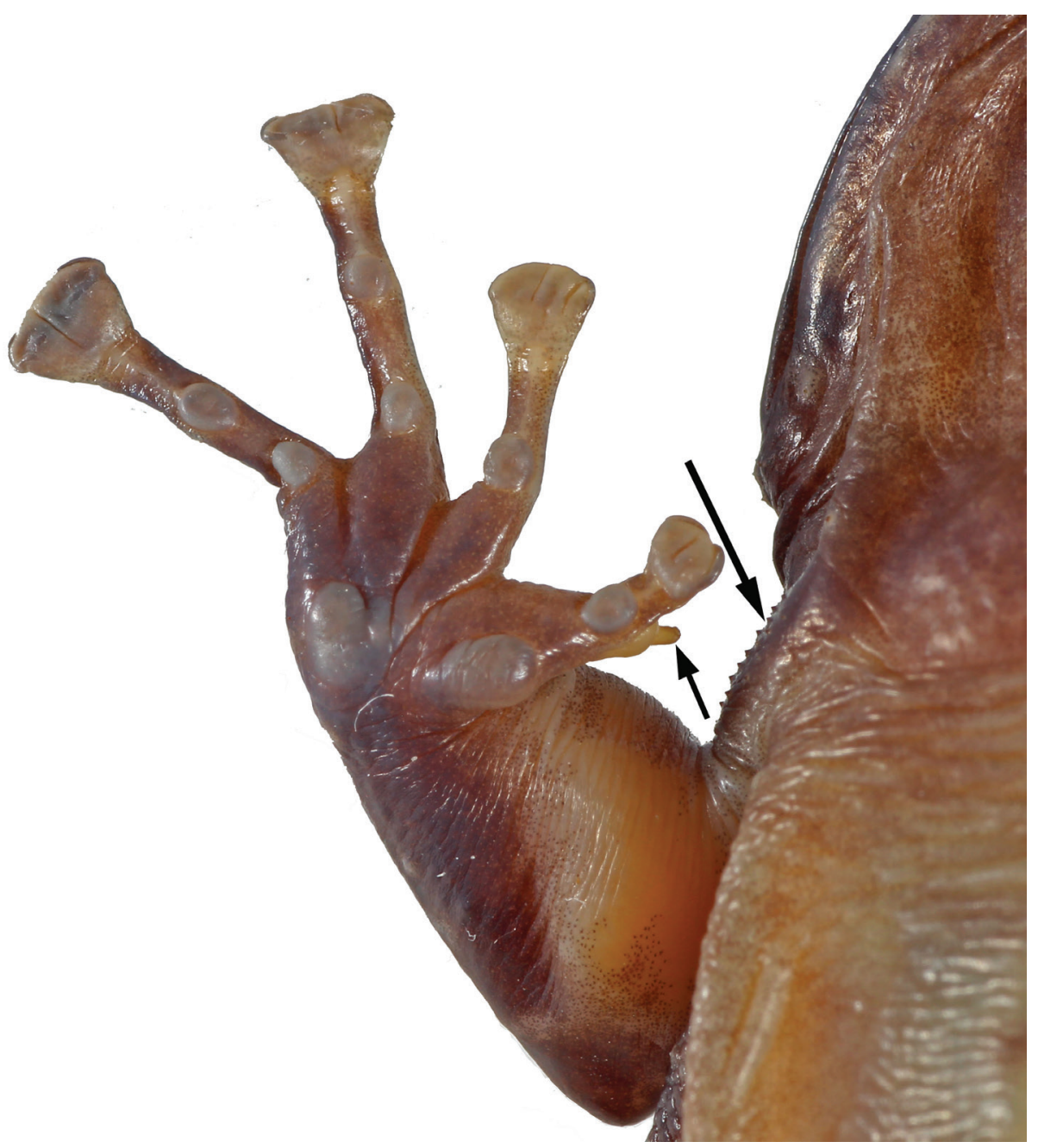

Figure 9. Ventral view of the right forelimb of Petropedetes newtonii (adult male, MNCN 48728). Arrows point to the dorsal spine on the distal edge of the metacarpal of Finger I and keratinised spicules along the skin surface of the upper arm and labial commissure.

$40.3 \%$ of head length; interorbital distance $2.4 \mathrm{~mm}$; loreal region highly concave; tympanum distinct, rounded, not flattened in its upper side; tympanic papilla present, located in the upper margin of tympanum; supratympanic fold distinct, extending from behind the eye, bordering the tympanum, to close the level of shoulder; choanae moderately large, subcircular; vomerine teeth present, relatively big and close to each other, transversally positioned between choanae and eye orbit; tongue elongated, oval, cordiform (heart-shaped, notched posteriorly) and with a single papilla located in the middle of the anterior region.

Forelimb robust; forearm hypertrophied; paired humeral crest high, extending over most of humerus length; relative lengths of fingers III > IV > II > I; palmar webbing absent; tips of fingers flat, expanded as adhesive discs; adhesive discs heart-shaped, with 
two oval plates on the dorsal side; relative width of terminal discs IV $=$ III $>$ II $>$ I; terminal phalanges T-shaped; thenar (inner palmar) tubercle oval, more than $1 / 3$ of Finger I total length; outer palmar tubercle distinct, rounded, bigger than thenar tubercle; one subarticular tubercle on Finger I, placed between fingertip and the centre of the finger; subarticular tubercle of Finger II centrally positioned; fingers III and IV with two subarticular tubercles; dorsal spine on the distal edge of the metacarpal of Finger I robust, whitish; keratinised spinules on humeral skin present. Hind limbs moderately robust and long (femur and tibia length $42.1 \mathrm{~mm}$ ); femoral gland large, subcircular, $33.5 \%$ of femur length; toes not webbed or rudimentarily; tips of toes flat, expanded as adhesive discs; adhesive discs heart-shaped, with two oval plates on the dorsal side; relative width of terminal discs: II > I > III > IV > V; terminal phalanges T-shaped; toes long; relative length of toes: IV > III > V > II > I; toes I and II with one single tubercle, toes III and V with two single tubercles and Toe III with three single tubercles; no supernumerary tubercles; outer metatarsal tubercle absent; a distinct, elongated inner metatarsal tubercle.

Skin of dorsum with scattered pustules, especially distinct in the anterior region at the level of the shoulder; keratinised spicules on inner surface of upper arm, lower tympanic region, supratympanic fold, and on postcommissural region; ventral skin smooth.

Coloration of dorsal surfaces in preservative dominated by different brown tonalities, with whitish transversal lines or marks dispersed on hind and fore limbs; posterior margin of finger and toe tips whitish. Ventral coloration white, except in the throat, palmar surfaces, and tibia, which are brownish; inner side of forearms white, external side brown; ventral side of hind legs whitish with dispersed, brown rounded spots.

\section{Distribution of Petropedetes newtonii}

There are few geographical records published of $P$. newtonii sensu stricto. The original type locality lacks a specific location in Bioko; however, the second known specimen was collected in Basilé, Bioko Norte province ("Bassile", at $527 \mathrm{~m}$ asl. on the grass [Bocage 1895b]). Posteriorly, other populations of P. newtonii were recorded within Bioko Sur province: Musola (Boulenger 1906), Rio Iladyi, at $1000 \mathrm{~m}$ asl. (Mertens 1965), and another in Bioko Norte province without specific locality: NW Bioko (Bocage 1903). Barej et al. (2014) provided the following specific localities: Río Osa, Río Ole, Río Sibitá and Caldera de Luba, based on specimens held at the Museo Nacional de Ciencias Naturales, all collected by SC-F in 2003 (Table 1). Additonally, Barej et al. (2014) studied populations from the southern coast of Cameroon, which are nested within the clade of Biokoan populations of P. newtonii (considered as P. vulpiae by Barej et al. [2014]).

Therefore, populations from continental Africa like those from Río Muni (Equatorial Guinea), that were formerly considered as P. newtonii (De la Riva 1994, Lasso et al. 2002) are now included in the taxon P. vulpiae (Barej et al. 2010). The distribution of $P$. newtonii would be formed by widely distributed populations throughout Bioko (both in Bioko Norte and Bioko Sur) and by continental populations on the southern coast of Cameroon (Fig. 10). 
As a consequence of this validation, it must be said that the tadpole of $P$. newtonii has been described by Barej et al. (2010) (as P. johnstoni) based on a specimen from Musola, Bioko, which was cited by Boulenger (1906).

Natural history. Descriptions are based on the field notes of SC-F (Table 1). All specimens were collected after dusk between 18:45 and 19:30, the furthest distance from the water $2 \mathrm{~m}$, in the shallower parts $(20 \mathrm{~cm}$ depth) of streams between 22 November and 03 December 2003, which typically corresponds to the rainy season. The exceptions are three juveniles (MNCN 48960, 48962, and 48958) found during the day, one of them (MNCN 48960 ) in a place with no body of water in the immediate surroundings (Table 1). Two adult specimens (male MNCN 48730 and gravid female MNCN 48955) collected on a mid-elevation affluent of the right margin of the Olé River $\left(3^{\circ} 18^{\prime} 27.08^{\prime \prime} \mathrm{N}, 8^{\circ} 28^{\prime} 24.36^{\prime \prime E}\right)$ were found on rocks, either in the middle of the stream (the female) or on the shore (the male). The juvenile MNCN 48962 was also found on the moss covering a rock in a stream. Most of the other specimens were found on the upper side of medium-sized leaves, 7-35 $\mathrm{cm}$ above the water or ground levels. The adult female MNCN 48956 was perched on a thin branch (1 cm diameter) $1.80 \mathrm{~m}$ above the water, while juveniles MNCN 48958 and 48960 were found on the ground. No specimen was observed vocalising or engaging in reproductive behaviours such amplexus, egg deposition, or providing care to offspring.

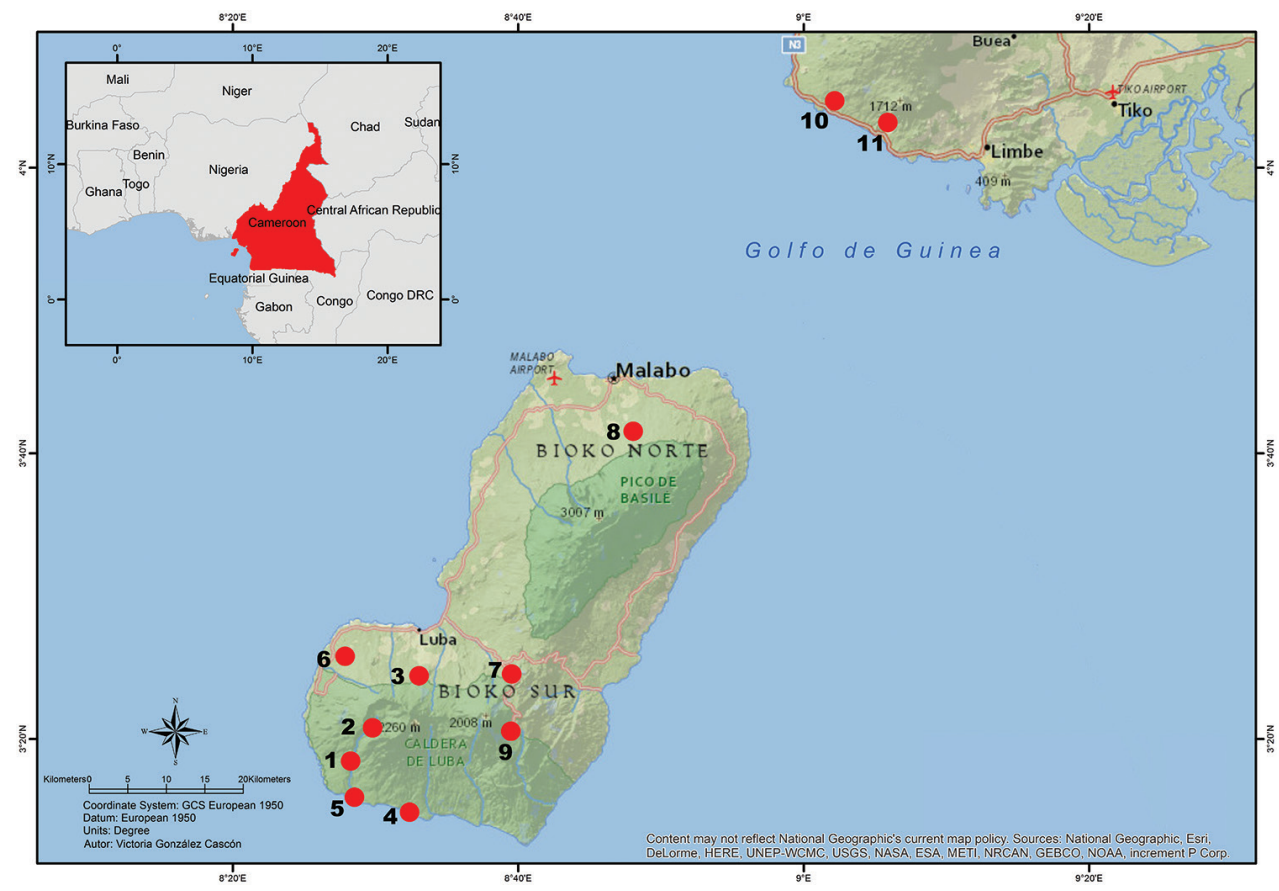

Figure 10. Distribution map of Petropedetes newtonii based on published records and collection data. I Campamento Smith, Luba, Bioko 2 Río Riaco, Caldera de Luba, Bioko 3 Stream Mukokobe, between Belebu and Ureka, Bioko 4 Río Osa, Bioko 5 Río Ole, Bioko 6 Río Sibitá, Bococo, Bioko 7 Musola, Bioko 8 Basilé, Bioko 9 Río Iladyi, Bioko 10 Bakingili, Cameroon I I Mt. Etinde, Cameroon. 
Key to adult Petropedetes species, modified from Barej et al. (2010) papillae and dorsal spine on the distal edge of the metacarpal of Finger I, tympanum in females up to $3 / 4$ of eye diameter, femoral glands large........P. perreti Tympanum small, indistinct in both sexes, males without tympanic papillae, but with dorsal spine on the distal edge of the metacarpal of Finger I, femoral glands large to very large

P. palmipes

Toes half-webbed

Tympanum distinct, males with tympanic papillae and dorsal spine on the distal edge of the metacarpal of Finger I, femoral gland line shaped in both sexes

P. juliawurstnerae

Tympanum indistinct, males without tympanic papillae, dorsal spine on the distal edge of the metacarpal of Finger I absent, femoral gland ovoid

P. cameronensis

Tympanum small, rounded, in males smaller than eye diameter, tympanic papillae close to upper border of tympanum, femoral glands large

Males with dorsal spine on the distal edge of the metacarpal of Finger I present, keratinised spicules on arms and tympanic borders present, large thenar tubercle, male humeral crest well developed, reaching more than half of the total humeral length, snout slightly pointed.

P. newtonii

Males with metacarpal spines absent, keratinized spicules on arms and tympanic borders absent, relatively small thenar tubercle, humeral crest reaching the half of the total length of the humerus, snout slightly rounded.....

P. johnstoni Femoral gland of moderate size, tympanum in males usually flattened with tympanic papillae closer to the centre than to upper border, dorsal spine on the distal edge of the metacarpal of Finger I present in males

P. vulpiae Femoral gland small, tympanic papillae closer to upper border than to the centre, dorsal spine on the distal edge of metacarpal of Finger I present ......8 Femoral gland small, in males $22 \%$ of femur length, tympanum size variable from $3 / 4$ of the eye diameter to larger than the eye, lowland species .... P. parkeri Femoral gland very small, in males $16 \%$ of femur length, tympanum in males as large as eye diameter, highland species. 


\section{Acknowledgements}

Fieldwork in Bioko by SC-F was possible thanks to the Asociación Amigos de Doñana $(\mathrm{AAD})$ and its director Javier Castroviejo. While in Bioko, the help of Ramón Castelo (AAD) proved invaluable. We are grateful to Mario García-París for his valuable comments and to Arlo Hinkley for his English revision of the manuscript. We appreciate the kindness of Ignacio Martín for depositing in the MNCN collection specimens of several frog species collected in Bioko. We thank Ursula Bott from the Zoologisches Forschungsmuseum Alexander Koenig, Bonn (ZFMK), who kindly sent material on loan. Also, our gratitude to Cristina Paradela for generating the CT-scan image. We thank Victoria González for kindly making the map illustration. Finally, we thank Annemarie Ohler and Andreas Schmitz for their helpful reviews of the manuscript.

\section{References}

Amiet J-L (1983) Une espèce méconnue de Petropedetes du Cameroun: Petropedetes parkeri n. sp. (Amphibia Anura: Ranidae, Phrynobatrachinae). Revue Suisse de Zoologie 90: 457-468. https://doi.org/10.5962/bhl.part.81986

Barej MF, Rödel MO, Gonwouo LN, Pauwels OSG, Böhme W, Schmitz A (2010) Review of the genus Petropedetes Reichenow, 1874 in Central Africa with the description of three new species (Amphibia: Anura: Petropedetidae). Zootaxa 2340: 1-49.

Barej MF, Rödel MO, Loader SP, Menegon M, Gonwouo NL, Penner J, Gvoždík V, Günther R, Bell RC, Nagel P, Schmitz A (2014) Light shines through the spindrift - Phylogeny of African torrent frogs (Amphibia, Anura, Petropedetidae). Molecular Phylogenetics and Evolution 71: 261-273. https://doi.org/10.1016/j.ympev.2013.11.001

Bocage JVB (1895a) Sur un batracien nouveau de Fernão do Pó. Jornal de Sciências Matemáticas, Fisicas e Naturais, Academia das Sciências de Lisboa; Segunda Série 3: 270-272.

Bocage JVB (1895b) Reptiles et Batraciens nouveaux ou peu connus de Fernão do Pó. Jornal de Sciências Matemáticas, Fisicas e Naturais, Academia das Sciências de Lisboa; Segunda Série 4: 16-20.

Bocage JVB (1903) G.A. Boulenger - Batraciens nouveaux et Reptiles nouveaux. Jornal de Sciências, Mathemáticas, Physicas e Naturaes Lisboa 7(2): 62-64.

Boulenger GA (1888) A list of the reptiles and batrachians collected by Mr. H. H. Johnston on the Rio del Rey, Cameroons District, W. Africa. Proceedings of the Royal Society of London 1888: 564-565.

Boulenger GA (1900) A list of the batrachians and reptiles of the Gaboon (French Congo), with descriptions of new genera and species. Proceedings of the Zoological Society of London 1900: 433-456.

Boulenger GA (1906) Report on the batrachians collected by the late L. Fea in West Africa. Annali del Museo Civico di Storia Naturale di Genova 42: 157-172. 
De la Riva I (1994) Anfibios anuros del Parque Nacional de Monte Alén, Río Muni, Guinea Ecuatorial. Revista Espańola de Herpetología 8: 123-139.

De la Riva I, Trueb L, Duellman WE (2012) A new species of Telmatobius (Anura: Telmatobiidae) from montane forests of southern Peru, with a review of osteological features of the genus. South American Journal of Herpetology 7: 91-109. https://doi.org/10.2994/057.007.0212 Frétey T, Dewynter M, Blanc C (2012) Amphibiens d'Afrique centrale et d'Angola - Clé de détermination illustrée des amphibiens du Gabon et du Mbini. Biotope, Mèze (Collection Parthénope); Muséum national d'Histoire naturelle, Paris, 232 pp.

Frost DR (2018) Amphibian Species of the World: an Online Reference. Version 6.0 (12/03/2018). Electronic Database accessible at http://research.amnh.org/herpetology/ amphibia/index.html

Mertens R (1965) Die Amphibien von Fernando Poo. Bonner zoologische Beiträge 16: 14-29.

Mertens R (1968) Zur Kenntnis der Herpetofauna von Kamerun und Fernando Poo. Bonner zoologische Beiträge 19: 69-84.

Lasso CA, Rial AI, Castroviejo J, De la Riva I (2002) Herpetofauna del Parque Nacional de Monte Alén (Río Muni, Guinea Ecuatorial). Graellsia 58: 21-34. https://doi.org/10.3989/ graellsia.2002.v58.i2.276

Parker HW (1936) The amphibians of the Mamfe Division, Cameroon. (1) Zoogeography and systematics. Proceedings of the Zoological Society of London 1936: 135-163. https://doi.org/10.1111/j.1096-3642.1936.tb02284.x

Perret J-L (1976) Revision des amphibiens africains et principalement des types, conservés au Musée Bocage de Lisbonne. Arquivos do Museu Bocage 6: 15-34. 